\title{
Alcohol-induced changes in contrast sensitivity following high-intensity light exposure
}

\author{
ANTHONY J. ADAMS \\ School of Optometry, University of California, Berkeley, California 94700 \\ BRIAN BROWN \\ Smith-Kettlewell Institute of Visual Sciences, San Francisco, California 94115 \\ and \\ MERTON C. FLOM \\ School of Optometry, University of California, Berkeley, Califormia 94700
}

\begin{abstract}
Relatively low doses of alcohol produced large, significant, dose-related increases in the time required to recover foveal contrast sensitivity following bright light exposure. Nine subjects participated in a double-blind experiment involving three dose levels of alcohol (including placebo). The luminance parameters of the test were comparable to those encountered in practical situations such as driving. The alcohol-induced delay in glare recovery is probably retinal and lasts for several hours after drinking.
\end{abstract}

The eye has a relatively fixed contrast sensitivity over a wide range of normal daytime light levels. However, sudden changes in the environmental light level require the eye to readjust in order to achieve the same contrast sensitivity at the new level, a process which takes many seconds or minutes when the new environment is considerably dimmer than the previous one. During this recovery time, the eye is relatively blind to fine detail, its contrast sensitivity being reduced while it adapts. The initial stages of recovery are rapid and generally thought to involve neural processes in the retina (Baker, 1963), whereas the predominant slow phase is related to the rate of regeneration of the bleached photopigment.

When the integrity of the retina is disturbed by drugs or disease, the adaptation process is retarded and the recovery of sensitivity may be dangerously prolonged. Certain drugs and retinal diseases are reported to prolong the recovery of sensitivity without an accompanying loss of clinically measured Snellen visual acuity (Carr, Henkind, Rothfield, \& Siegel, 1968; Severin, Tour, \& Kershaw, 1967). More recently, the anesthetic halothane has been shown to act directly on the retina to prolong cone dark adaptation, an effect which is interpreted as due to changes in cone photopigment kinetics (Norren \& Padmos, 1975).

Alcohol is also known to have a direct effect on the retina. Ikeda (1963) and Jacobson, Hirose, \& Stokes

We thank Dr. Alex Cogan, Dr. Gunilla Haegerstrom-Portnoy, George Mertz, and Michael Muegge for assistance with this project. This research was supported by Contract No. DADA 17-73-C-3106 from the U.S. Army Medical Research and Development Command to the Visual Sciences Division of the Optical Sciences Group, San Rafael, California.
(1969) showed that the $b$ wave of the human dark-adapted electroretinogram was altered by alcohol levels commonly encountered in social drinking. Skoog (1974) showed that the $c$ wave was also altered by alcohol. Raskin, Sligar, and Steinberg (1973) reported an alcohol-induced slowing of dark adaptation in rats, with direct evidence that this parallels a reduced rate of resynthesis of visual pigments. In spite of these findings, most commonly measured visual functions are generally considered resistant to alcohol. For example, Adams, Brown, Flom, Jones, and Jampolsky (1975) have shown that visual acuity, even at low contrast levels, is unaltered by alcohol doses identical to those reported in our present study.

Human psychophysical experiments have yielded apparently conflicting reports of the effect of alcohol on the time course of dark adaptation. Recovery was typically measured in the dark and required detection of a light flash or the resolution of a relatively large object. Some studies reported no effect of alcohol on the time course of dark adaptation (Colson, 1940; Blomberg \& Wassen, 1959; Giardini, 1948; Verriest \& Laplasse, 1965), others reported a slowing of the time course (Förster \& Starek, 1959; Newman \& Fletcher, 1941). In general, these studies were poorly controlled; the experiments were not run blind and dose-response relationships were not established. None of the studies involved the resolution of fine detail at photopic light levels.

In this double-blind experiment, we have shown that relatively low doses of alcohol produce large and significant dose-related increases in the time required to recover photopic foveal contrast sensitivity following bright-light exposure. 




Figure 1. The stimulus configuration of the $5.6 \times 10 \mathrm{~cd} / \mathrm{m}^{2}$ glare field on the keft and the test field on the right. The 5-min-of-arc test spot is located at the center of the test field reference markers on a background of luminance $22.6 \mathrm{~cd} / \mathrm{m}^{2}$.

\section{METHOD}

Nine young male subjects (aged from 20 to 28 ) participated in a replicated 3 by 3 crossover experiment which was run double-blind. Two alcohol doses $(0.5$ and $1.0 \mathrm{ml} / \mathrm{kg}$ body weight) and a placebo were used. Alcohol in the form of $95 \%$ ethanol was diluted with fruit juice so that the total volume (milliliters) was 3 times the subject's body weight (kilograms). The drink was consumed through a straw from a lidded paper cup which also contained two ice cubes. To minimize olfactory cues to the presence of alcohol, 2 drops each of ethanol and eucalyptus were placed on the lid of the cup. Twenty minutes were allowed for the consumption of the drink. Blood alcohol level (BAL) was estimated before drinking and at intervals after drinking using the Intoxilizer (Omicron Systems. Palo Alto), which estimates BAL using infrared spectroscopy of a breath sample.

In each measurement session, subjects preadapted for at least $5 \mathrm{~min}$ to the low photopic luminance levels of the test laboratory. Fixation was aided by four thin diagonally oriented reference lines. The subject fixated the center of a $20^{\circ}$ circular ad apting field of $5.6 \mathrm{x}$ $10 \mathrm{~cd} / \mathrm{m}^{2}$ with the right eye (see Figure 1). Immediately following a 10-sec exposure to the high intensity adapting field, the subject fixated the center of the stimulus configuration where a test spot (5 min of arc) was intermittently presented $(125-\mathrm{msec}$ flashes at $4 \mathrm{~Hz}$ ) on a $22.6-\mathrm{cd} / \mathrm{m}^{2}$ background (Figure 1). The contrast of the test spot was under the subject's control. When he had recovered contrast sensitivity to a fixed contrast, he turned a switch to reduce the target contrast a further fixed step below his threshold. When his contrast sensitivity had again recovered to the point of detection, he again turned the switch and the spot contrast was further stepped down. The times at which the switch was operated were recorded, and in this way the time taken to recover to each of five predetermined contrast levels $(5.34,2.00,1.45,0.99$, and 0.64$)$ was obtained. These were chosen to give approximately equal time periods between each recovery point; contrast was always the independent variable. Four sets of measurements were taken, with 5 min allowed between sets for complete recovery from the adapting luminance level. The intertest interval was based on repeated empirical measures of four subjects prior to beginning the experiment. The adapting source, the test spot, and the background were initially calibrated using a Macbeth illuminometer; luminance levels were checked on each test day using built-in phototransistors. Further periodic direct luminance checks were made with the Macbeth illuminometer. The stimulus and response events were recorded on a Beckman polygraph for later measurement.

Photographs of the left eyes of five subjects were taken $5 \mathrm{sec}$ before and $5 \mathrm{sec}$ into exposure to the high-intensity adapting stimulus during the first trial of each test period. Measurements of pupil size were made from the projected negatives.
Subjects practiced the task to achieve stable performance before participation in the experimental sessions. On a given experimental day, they were tested before drinking and 30,90, 180, 270. and 360 min after drinking. At each of these test times. blood alcohol levels and subjective intoxication ratings were ascertained by a second experimenter. (Subjects rated their high level on a scale of 0 to 100 , where 0 was sober and 100 was as intoxicated as the subject had ever been on alcohol.)

\section{RESULTS}

\section{Recovery Time}

The time course of adaptation following the 10-sec high-intensity exposure was significantly retarded for both the 0.5 and $1.0 \cdot \mathrm{ml} / \mathrm{kg}$ doses of alcohol $90 \mathrm{~min}$



Figure 2. Glare recovery time as a function of $\log$ target contrast for placebo and alcohol $90 \mathrm{~min}$ after drinking. $1.0 \mathrm{ml} / \mathrm{kg}$ body weight of $95 \%$ ethanol $(O), 0.5 \mathrm{ml} / \mathrm{kg}$ body weight of $95 \%$ ethanol $(\Delta)$, and placebo $(\square)$. Mean predrink values for all subjects collaps $\%$ across test days are also shown $(x)$. The dose relationship for the alcohol doses is evident in the displacement of the two alcohol treatment curves. 
Table 1

Number of Subjects (Out of Nine) With Longer Adaptation Time at 90 Min After Drinking Than Before Drinking

\begin{tabular}{lccccc} 
& \multicolumn{5}{c}{ Contrast Level } \\
\cline { 2 - 6 } Drug Condition & 5.34 & 2.00 & 1.45 & .99 & .64 \\
\hline Placebo & 5 & 5 & 6 & 4 & 5 \\
$.5 \mathrm{ml} / \mathrm{kg}$ Alcohol & 7 & 9 & 9 & 8 & 7 \\
$1.0 \mathrm{ml} / \mathrm{kg}$ Alcohol & 9 & 7 & 8 & 7 & 8 \\
\hline
\end{tabular}

Table 2

Significance Levels for Changes in the Recovery Functions of Figure 3 (Friedman Two-Way Analysis of Variance)

\begin{tabular}{llllll} 
& \multicolumn{5}{c}{ Contrast Level } \\
\cline { 2 - 6 } \multicolumn{1}{c}{ Drug Condition } & 5.34 & 2.00 & 1.45 & .99 & .64 \\
\hline Placebo & n.s. & n.s. & n.s. & n.s. & n.s. \\
$.5 \mathrm{ml} / \mathrm{kg}$ Alcohol & n.s. & .01 & n.s. & .05 & n.s. \\
$1.0 \mathrm{ml} / \mathrm{kg}$ Alcohol & .001 & .01 & .001 & .01 & .001 \\
\hline
\end{tabular}

after drinking when blood alcohol levels were near their peak. Figure 2 shows the group recovery time to test spots of different contrast for placebo and 0.5 and $1.0 \mathrm{ml}$ of $95 \%$ ethanol $/ \mathrm{kg}$ body weight at this time. The recovery times for both alcohol doses were all signicantly longer than the corresponding predrink values $(p<.02$. Walsh test). There was a relatively large increase in group variance with time after drinking due largely to the individual differences in response to the alcohol dose. However, the variance for most subjects showed only a small increase after the alcohol treatment. Table 1 indicates the number of subjects whose recovery time was increased by each of the experimental conditions.

Group mean recovery times are longer by between $30 \%$ to $50 \%$ for the $1.0 \mathrm{ml} / \mathrm{kg}$ alcohol dose compared to the corresponding placebo values. It is apparent

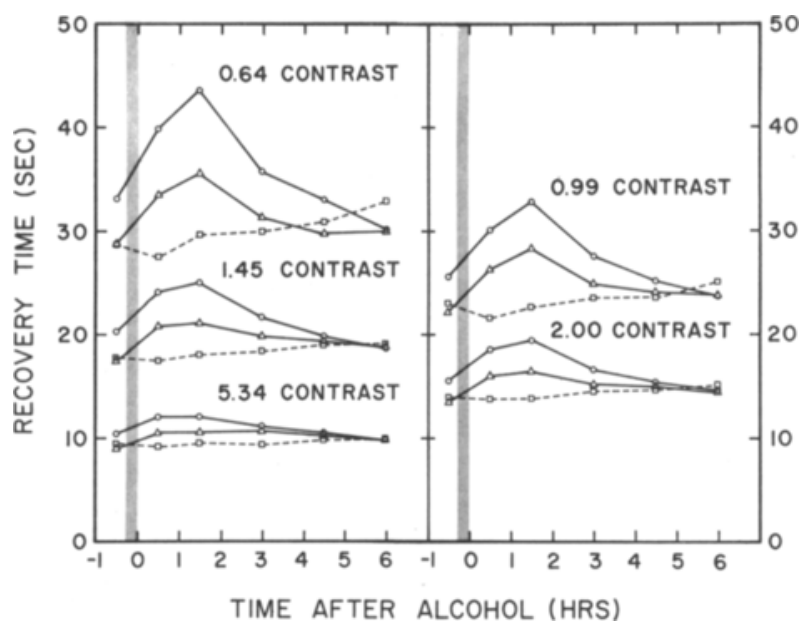

Figure 3. Time course of glare recovery to tangets of contrasts $5.34,2.00,1.45,0.99$, and 0.64 after ingestion of alcohol. $1.0-\mathrm{ml} / \mathrm{kg}$ body weight of $95 \%$ ethanol $(0), 0.5 \mathrm{ml} / \mathrm{kg}$ body weight of $95 \%$ ethanol $(\Delta)$, and placebo $(\square)$. The shaded area indicates the time of alcohol ingestion.
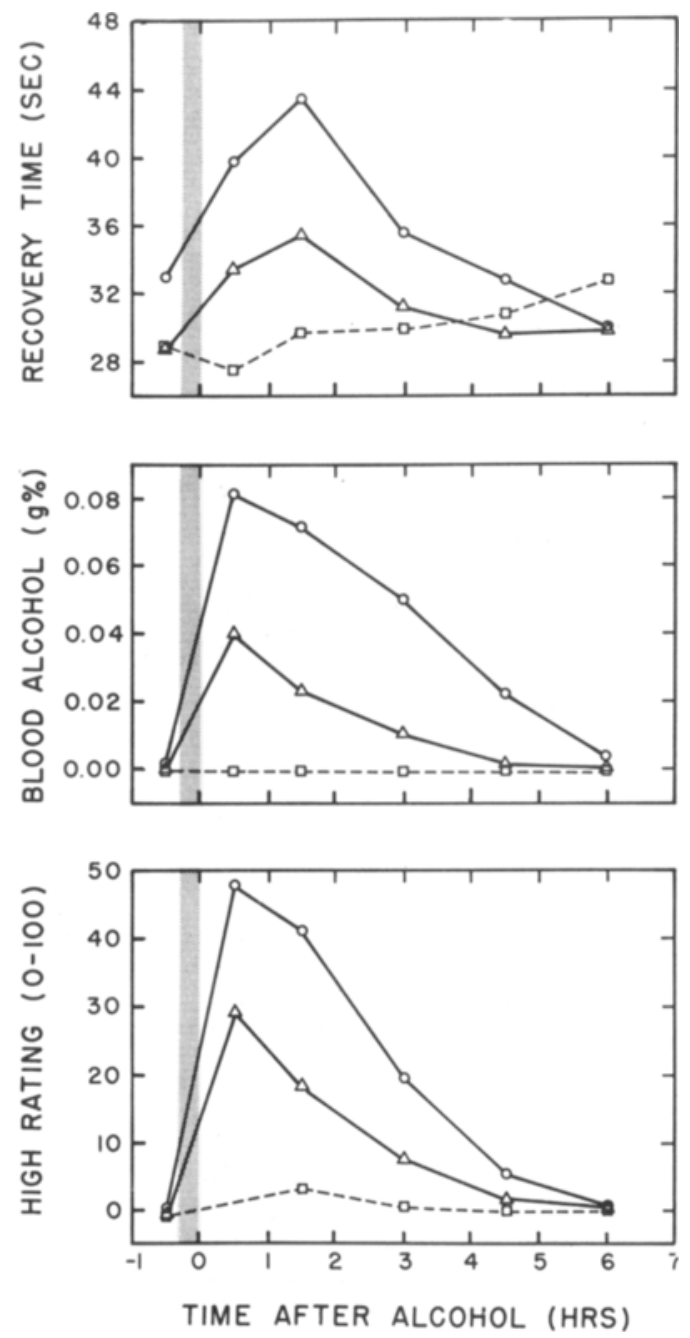

Figure 4. Time course after alcohol Ingestion of mean foveal recovery time (seconds) for the lowest centrast $(0.64)$, blood alcohol level, and subjective high rating. $1.0 \mathrm{ml} / \mathrm{kg}$ body weight of $95 \%$ ethanol $(O), 0.5 \mathrm{ml} / \mathrm{kg}$ body weight of $95 \%$ ethanol $(\triangle)$, and placebo ( $\square)$. The shaded area indicates the time of alcohol ingestion.

from Figure 2 that the contrast thresholds at any given contrast during the recovery phase are almost twice as high ( 0.25 to $0.3 \log$ units) as the predrink thresholds. The insensitivity of the measure to placebo treatment is reflected in Figure 2; the mean predrink recovery times fall on the curve generated by the placebo recovery times.

\section{Glare Recovery Decrement: Time Course of Alcohol Effect}

For all contrasts, the alcohol-induced increase in glare recovery time was apparent in the first measurement period $(30 \mathrm{~min})$, peaked at $1-2 \mathrm{~h}$, and subsequently declined to reach predrink values approximately $6 \mathrm{~h}$ after drinking (see Figure 3).

A Friedman two-way analysis of variance, which 


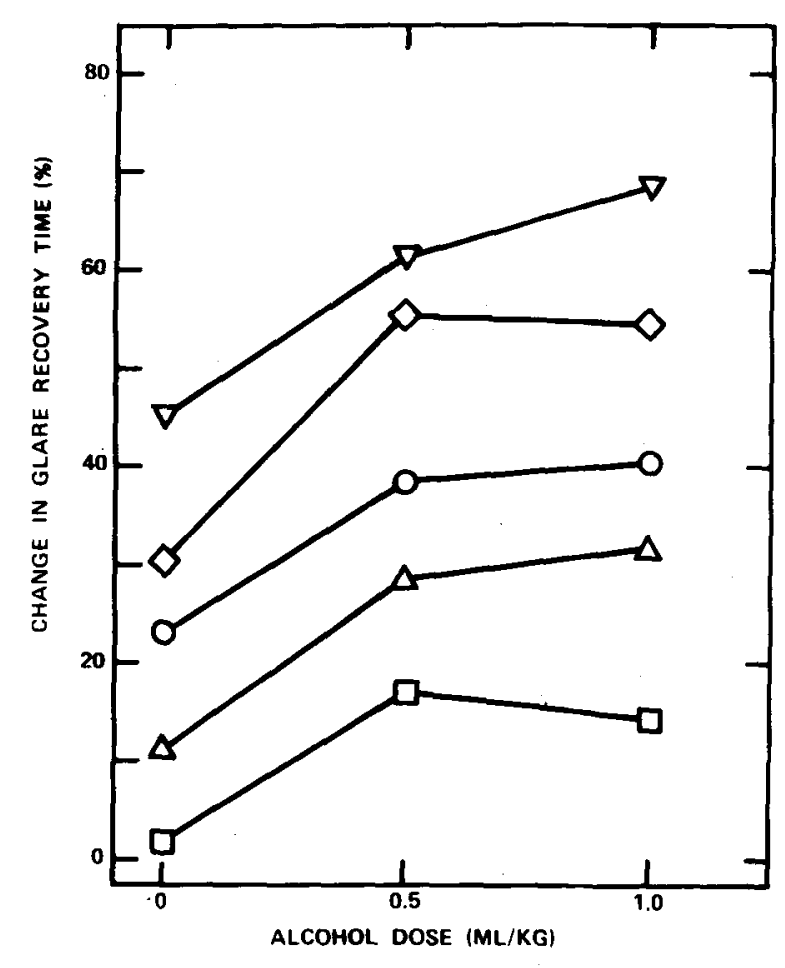

Figure 5. Mean percent change in glare recovery time at $90 \mathrm{~mm}$ from prelevek in glare recovery time for nine subjects as a function of alcohol dose. Contrast levels: $5.34(\square), 2.00(\Delta), 1.45(0), 0.99$ $(())$, and $0.64(\nabla)$. Each successive curve has been arbitrarily displaced upwards by $10 \%$ for the sake of clarity.

tests for systematic changes in recovery time, was performed for each of the curves in Figure 3, resulting in significance levels as shown in Table 2.

There is a significant systematic reduction in glare recovery time at all five contrast levels for the high dose of alcohol $(1.0 \mathrm{ml} / \mathrm{kg})$ and for the two contrast conditions on the right of Figure 3 for the low dose of alcohol $(0.5 \mathrm{ml} / \mathrm{kg})$.

There are impressive parallels in the time course of the blood alcohol levels, subjective high ratings and the time taken to recover from the high-intensity light exposure. It can be seen from Figure 4 that the three measures are similar in time course.

However, the peak of the recovery-time function appears to be delayed slightly when compared to blood alcohol levels and high ratings. This raises the interesting possibility that the increases in recovery time may be produced by the action of an intermediate product of alcohol metabolism, such as acetaldehyde, which has a delayed time course compared to blood alcohol (Korsten, Matsuzaki, Feinman, \& Lieber, 1975).

\section{Dose Relationship}

The alcohol-induced increases in recovery times are dose related. The dose relationship was clearly evident 90 min after drinking when there was a maximum retardation in the recovery of contrast sensitivity for the group at each contrast level (Figure 5). Recovery times were delayed by an average of $20.9 \%$ for the $0.5-\mathrm{ml} / \mathrm{kg}$ dose and $24.4 \%$ for the $1.0-\mathrm{ml} / \mathrm{kg}$ dose. Furthermore, it is evident from Figure 3 that a dose relationship existed for at least $3 \mathrm{~h}$ after drinking.

Each individual recovery time has a blood alcohol measure associated with it. When all such paired measures are considered across subjects, drug conditions, and times before and after drug ingestion. the relationship between blood alcohol level and glare recovery time can be established. Such relationships are shown for each target contrast in Figure 6.

The relatively large increase in recovery times for low blood alcohol levels is impressive and reflects the high sensitivity of this visual function to alcohol.

\section{Role of Preretinal Factors}

Alcohol induced changes in pupil size could possibly influence the recovery time to a fixed contrast; a larger pupil would increase the total light reaching the retina during the high-intensity exposure while perhaps slightly decreasing the sharpness of the retinal image of the 5-min arc test target. In order to examine the role of the pupil, the diameter of the pupil was measured photographically prior to bright light exposure as well as midway through the exposure ( $5 \mathrm{sec}$ after light onset) on five of the subjects. The pupil size thus measured in each condition changed very little, and there were no consistent trends in pupil size either over time after drinking or for different

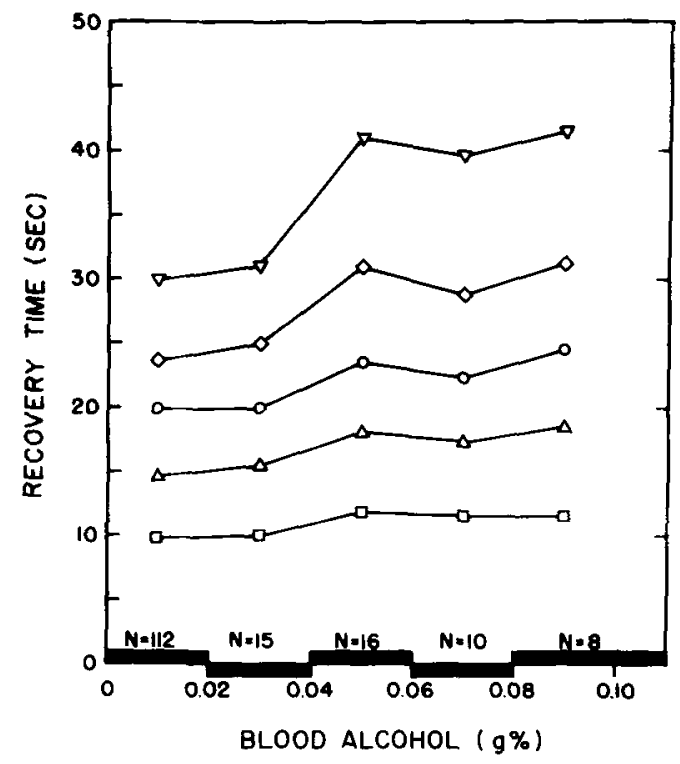

Figure 6. Mean recovery time to targets of five contrasts as a function of blood alcohol level. The range of blood alcobol levels was divided into five equal intervals, and the corresponding glare recovery times were averaged within these intervals $(0$ to .019 , $.02-.039, .04-.059, .06-.079, .089 \%$ and greater) aliong the abscissa. Contrast levebs: $5.34(D), 2.00(\Delta), 1.45(O), 0.99(())$, and 0.64 $(\nabla)$. 
doses. Figure 7 shows the results for a subject whose glare recovery times were markedly altered by alcohol at all contrast levels. Both the recovery times and the pupil diameters measured during and before the glare are presented for the $1.0-\mathrm{ml} / \mathrm{kg}$ dose. Ninety minutes after drinking, the pupil sizes before and during glare exposure were the same as the predrink measures; at the same time, there was an average increase in glare recovery time across contrast levels of $54 \%$ for the subject. Moreover, during the last $3 \mathrm{~h}$, when recovery times were elevated and returning to predrink levels, pupil size was constant. It was no larger than at $30 \mathrm{~min}$ after drinking when recovery times were most prolonged. In a further attempt to estimate the way in which changes in pupil size alone could have influenced recovery time, we dilated the pupil of an additional nondrug subject with 1 drop of $1 \%$ Cyclogyl (cyclopentolate) and measured recovery times for the subject with the 8.5-mm-diam dilated pupil and through a 3 -mm-diam artificial pupil. In spite of an eightfold difference in pupil area, there was no significant difference in the time course of light adaptation (Figure 8).

Inexact focus on the test target, if it accompanied alcohol intoxication, also might produce longer recovery times. Our parafoveal reference markers (Figure 1) were designed to elicit fine control of



Figure 7. Recovery time (seconds) to five different contrasts as a function of time after alcohol; subject $J . W$. Contrast levels: 5.34 $(D), 2.00(\triangle), 1.45(O), 0.99(O)$, and $0.64(\nabla)$. Shown below is the pupil diameter (millimeters) for this subject before exposure to the glare field ( $\square$ ) and during exposure to the glare field $(\bullet)$ as a function of time after alcohol.



Figure 8. Glare recovery time (seconds) vs. log contrast for subject B.B. with $8.5-\mathrm{mm}$-diam dilated pupil $(\Delta), 3-\mathrm{mm}$-diam artificial pupil (D), as well as 3 -mm-diam artificial pupil with $+0.75 \mathrm{D}$ blur $(0)$.

accommodative focus and fixation. However, in order to estimate the magnitude of focusing error that would be required to produce significant change in recovery time, we measured recovery time in the same subject through +0.75 diopters of blur. These recovery times are also shown in Figure 8, which shows increased recovery times and a shift which was approximately equal to that seen for our group after the high alcohol $(1.0 \mathrm{ml} / \mathrm{kg})$ dose. This suggests that inexact focus could have contributed significantly to our results with alcohol. However, this amount of defocus produced a substantial blurring of the parafoveal markers in the test plane, a change which was never reported by the subjects in our alcohol experiments. Also, in separate experiments with 10 subjects, we have shown that visual acuity is unaltered by the alcohol levels we used (Adams et al., 1975); this finding essentially precludes the possibility that inexact focus accompanied the alcohol intoxication and produced the long recovery times.

\section{DISCUSSION}

In our experiments, relatively low doses of alcohol produced significant delays in glare recovery. Both the mechanisms of this alcohol-induced change in the adaptation process and the social consequences for practical tasks deserve attention.

\section{Mechanisms}

How does alcohol produce the increased recovery times? A number of preretinal factors could be involved. including pupil-size changes, pupil response to the adapting stimulus, accommodative error, and accuracy of eye fixation. Skoglund (1943) reported 
that alcohol dilates the pupil, while more recently Verriest and Laplasse (1965) showed that the pupil size under low photopic light levels $(3.5 \mathrm{ml})$, almost identical to those in our experiment, was unaltered by alcohol. Our own measurements show that alcohol did not affect pupil size and that glare recovery is relatively insensitive to changes in pupil size.

Inaccuracies of accommodation would reduce target contrast and thereby increase glare recovery times. It is unlikely that such alcohol-induced blurring of the target occurred, since none of our subjects reported blurring of the fixation reference target. Further, we have shown that visual acuity is unaffected by alcohol doses identical to those used in this study (Adams et al., 1975).

Inexact eye fixation following alcohol might cause the test target to be imaged on the peripheral retina where photopic contrast sensitivity is lower than at the fovea for small test targets (Aulhorn \& Harms, 1972). In our experiments, the reference markers (Figure 1) defined a central zone $1.3 \mathrm{deg}$ in diam, providing a strong "centration" reference to minimize inexact fixation. If unsteadiness of fixation was precipitated by the alcohol, then longer recovery times would be associated with substantially greater variance; in fact, only small changes in variance for most individuals accompanied the alcohol-induced increase in recovery times.

Alcohol has been shown to have a direct effect on the human retina. Using alcohol doses similar to ours, Ikeda (1963) showed that the dark-adapted human electroretinogram (ERG) is altered in ways that suggest a decrease in the amount of inhibitory interconnection in the retina. Raskin et al. (1973) found that alcohol.slowed dark adaptation and the resynthesis of photopigment in albino rats; they attributed these results to alcohol inhibiting the action of a catalyst required for the oxidative resynthesis of retinaldehyde from retinol. Central processes, although affected by alcohol, do not seem to be involved in the mechanisms of light and dark adaptation; the ERG, which is entirely a retinal response, reflects all of the changes of adaptation.

From the foregoing arguments and evidence, the mechanism for the observed alcohol-induced prolongation of glare recovery seems to be retinal. The results of Raskin et al. (1973) raise the interesting possibility that alcohol may be inhibiting the resynthesis of photopigment, a hypothesis that needs to be tested by measuring cone pigment kinetics.

\section{Practical Significance}

Regardless of the mechanism involved, the increased recovery time produced by alcohol intoxication must be viewed as critical from a practical point of view. The detection of both large and small objects is important for safe driving. While our laboratory experiments refer only to small objects, it is reasonable to assume that recovery of contrast sensitivity for large objects is similarly affected.

The period of recovery from glare is a period of relative blindness for the individual and is thus potentially hazardous. The sky may act as an extended glare source for the automobile driver, particularly soon after sunrise and just before sunset. The sky luminance levels under these conditions may be as high as those experienced by the subjects in our experiment (Hopkinson, 1954). Under certain circumstances, a driver will be forced to intermittently view very bright sky or be subjected to high luminance glare from light scattered by the windshield. Following the glare, important features of the driving environment are lost or less visible for recovery times of many seconds. Alcohol prolongs this recovery. The possible consequences of an additional $30 \%$ to $50 \%$ delay in seeing critical detail under driving conditions are obvious.

\section{REFERENCES}

Adams, A. J., Brown, B., Flom, M. C., Jones, R. T., \& JAMPOLSKY, A. Alcohol and marijuana effects on static visual acuity. American Joumal of Optometry and Physiological Optics, 1975, 52, 729-735.

Aulhorn, E., \& Harms, H. Visual perimetry. In E. Jameson \& L. M. Hurvich (Eds.), Handbook of sensony physiology (Vol. VII/4). Berlin \& New York: Springer-Verlag, 1972.

BAKER. H. D. Initial stages of dark and light adaptation. Journal of the Optical Society of America, 1963. 53. 98-103.

Blomberg, L. H., \& Wassen, A. Preliminary report on the effect of alcohol on dark adaptation, determined by an objective method. Acta Ophthalmologica. 1959, 37, 274-278.

Carr, R. E.. Henkind, P., Rothfield, N.. \& Siegel. I. Ocular toxicity of antimalarial drugs. Long term follow-up. American Journal of Ophthalmology, 1968, 66, 738-744.

Colson, Z. W. The effect of alcohol on vision. An experimental investigation. Journal of the American Medical Association, 1940, 115, 1525-1527.

Förster, B., \& Starck, H. J. Uber die Hell- und Dunkeladaptation unter Alkoholeinfluss. Deutsche Zeitschrift für Gesamte Gerichtliche Medezin, 1959, 49, 66-69.

Giardini, A. Ricerche sugli effetti della associazione alcoholanossia sul senso luminoso. Revista Medicina da Aeronautica (Rio de Janeiro), 1948, 11, 531-543.

Hopkinson, R. G. Measurements of sky luminance distribution at Stockholm. Journal of the Optical Society of America, 1954, 44. 455-459.

IKEDA, H. Effects of ethyl alcohol on the evoked potential of the human eye. Vision Research, 1963, 3, 155-169.

Jacobson. J. H., Hirose, T., \& Stokes, P. E. Changes in human ERG induced by intravenous alcohol. Ophthalmologica, 1969. 158, Suppl., 669-677.

Korsten, M. A., Matsuzaki, S., Feinman, L., \& Lieber, C. S. High blood acetaldehyde levels after ethanol administration. New England Journal of Medicine, 1975, 292, 386-389.

Newman, H., \& FleTCHER, E. The effect of alcohol on vision. American Journal of Medical Science, 1941, 202, 723-731.

NorRen, D. V., \& PADmos, P. Cone dark adaptation: The influence of halothane anesthesia. Investigative Ophthalmology, $1975,14,212-227$.

Raskin, N. H., Sligar, K. P., \& Steinberg, R. H. Dark adaptation slowed by inhibitors of alcohol dehydrogenase in the albino rat. Brain Research, 1973, 50, 496-500. 
Severin, S. L., Tour, R. L., \& Kershaw, R. H. Macular function and the photostress test 2. Archives of Ophthalmology, $1967,77,163-167$.

Skoglund, C. R. On the influence of alcohol on the pupillary light reflex in man. Acta Physiologica Scandinavica, 1943, 6, 94-96.

SKoog, K. The c-wave of the human D.C. registered ERG. III. Effects of ethyl alcohol on the c-wave. Acta Ophthalmologica, 1974, 52, 913-923.
Verriest, G., \& Laplasse, D. New data concerning the influence of ethyl alcohol on human visual thresholds. Experimental Eye Research, 1965, 4, 95-101.

\footnotetext{
(Received for publication August 29, 1975; revision received November $28,1975$.
}

\section{ERRATUM}

Moskowitz, H. R., Dravnieks, A., \& Klarman, L. A. Odor intensity and pleasantness for a diverse set of odorants, 1976, 19(2), 122-128-This article was paginated incorrectly: Page 122 is correct. However, page 123 should be page 124; page 124 should be page 125; page 125 should be page 126; page 126 should be page 127; and page 127 should be page 123. Page 128 is correct. Our apologies are extended to both the authors and the readers. 